\title{
LA EVALUACIÓN DE COMPETENCIAS INTERPERSONALES EN LA FORMACIÓN DEL PROFESORADO DE EDUCACIÓN FÍSICA A TRAVÉS DE UN PROGRAMA DE EXPRESIÓN CORPORAL
}

\author{
(THE ASSESSMENT OF INTERPERSONAL COMPETENCES IN THE INITIAL TEACHING \\ TRAINING IN PHYSICAL EDUCATION THROUGH A PROGRAM OF BODY EXPRESSION)
}

Jose LUIS Aparicio HeRguedas, ANTONIO FraIle ARANDA 1 JOSELUIS.APARICIO.HERGUEDAS@UVA.ES; AFRAILE@MPC.UVA.ES

UNIVERSIDAD DE VALLADOLID

RESUMEN: Este artículo recoge cómo a través de un programa de expresión corporal, desarrollado en la formación inicial del profesorado de educación física con 47 estudiantes, se adquieren competencias docentes interpersonales que inciden en su mejora personal y profesional. Valorado el programa a través de una escala de valoración de competencias interpersonales del docente de educación física (EcoiDEF), consideran que este programa formativo les ha ayudado a mejorar su compromiso ético, el trabajo en equipo y las habilidades interpersonales.

Palabras clave: expresión corporal, formación inicial del profesorado de educación física, competencias interpersonales, dimensiones corporales, autoconocimiento.

ABSTRACT: This article collects how through a body expression program, developed in the initial training of teachers of physical education with 47 students, teaching skills that affect acquired in their personal and professional improvement. Evaluated the program through a rating scale of interpersonal skills in the teaching of physical education (EcoiDEF), considered that this training program has helped them improve their ethical commitment, teamwork, and interpersonal skills.

Keywords: body expression, initial teacher training in physical education, interpersonal competences, corporal dimensions, self-awareness.

\section{Introducción}

El modelo actual de sociedad del conocimiento exige a los educadores en general, y al profesorado de educación física en particular, proyectar y desarrollar un conjunto de competencias interpersonales $(\mathrm{Cl})$ con las que poder actuar personal y profesionalmente de forma más eficiente.

Las teorías socioconstructivistas de la Psicología Educativa enfatizan el papel activo del estudiante, siendo crucial que los docentes adquieran herramientas para responder a esa actividad, buscando con ello alcanzar mejoras en la autonomía, la participación y la autoeficacia (Vygotsky, 1978) de los futuros docentes en su proceso formativo.

En un estudio reciente realizado en Finlandia sobre competencias socioemocionales docentes (Talvio y otros, 2013) se aprecia que su desarrollo mejora tanto la gestión del aula como los grados de respeto y responsabilidad en el aprendizaje de los estudiantes. De la misma

\footnotetext{
${ }^{1}$ Dpto. Didáctica de la Expresión Musical, Plástica y Corporal. Facultad de Educación y Trabajo Social. Universidad de Valladolid. Paseo de Belén № 1, 47011. Valladolid (España).
} 
manera, se aprecian cambios positivos en la comunicación, en la toma de decisiones y en la autonomía de estudiantes y docentes.

La expresión corporal, en la actual sociedad de la información y del conocimiento, supone una burbuja en la que aún perviven valores básicos de convivencia y ambientes de aprendizaje en donde la libertad, el humor y el amor favorecen el desarrollo personal y social de los estudiantes (Montálvez y González López, 2012).

La expresión corporal pierde su esencia cuando se convierte en una actividad basada en la imitación 0 el entrenamiento. Se trata de un despertar a las sensaciones interiores para encontrar la unidad corporal y con ello ayudar a que los estudiantes alcancen un mejor autoconocimiento cognitivo, físico-corporal y emocional, entendiendo autoconocimiento como la capacidad de apartarse de nosotros mismos y examinar nuestra forma de pensar, nuestras acciones, nuestros hábitos y tendencias (Ojiro y Miralles, 2006).

Con este trabajo se pretende analizar qué $\mathrm{Cl}$ trabajadas a lo largo del programa de la asignatura Expresión y Comunicación Corporal son mas valoradas por los estudiantes y de qué forma se entienden a la luz de las capacidades que las estructuran.

\section{Contexto}

El estudio se ha realizado con la participación de 47 estudiantes (29 hombres y 18 mujeres) de $3^{\text {er }}$ curso de Educación Física (Grado de Maestro de Educación Primaria con la mención en Educación Física, MEPEF) que cursan estudios en la Facultad de Educación y Trabajo Social de la Universidad de Valladolid (UVa), durante el curso 2013-2014.

Los objetivos que se plantean para el presente estudio son: (1) conocer cuál es el grado de importancia que conceden los estudiantes universitarios de educación física a las $\mathrm{Cl}$ objeto de evaluación para su formación; (2) conocer de qué forma comprenden las $\mathrm{Cl}$ mejor valoradas a la luz de las capacidades que las estructuran.

\section{Descripción}

\subsection{La expresión corporal}

La asignatura Expresión y Comunicación Corporal forma parte del título universitario de Grado MEPEF que se imparte en la UVa y sus competenciaspropias se recogen en la ORDEN $\mathrm{ECl} / 3857 / 2007$. Si bien se prestará atención a todas y cada una de las competencias generales del título se trabajarán, de una manera significativa, las siguientes:

(1) Que los estudiantes hayan demostrado poseer y comprender conocimientos en un área de estudio, la Educación, que parte de la base de la educación secundaria general, y se suele encontrar a un nivel que, si bien se apoya en libros de texto avanzados, incluye también algunos aspectos que implican conocimientos procedentes de la vanguardia de su campo de estudio.

(2) Que los estudiantes sepan aplicar sus conocimientos a su trabajo o vocación de una forma profesional y posean las competencias que suelen demostrarse por medio de la elaboración y defensa de argumentos y la resolución de problemas dentro de su área de estudio, la Educación.

(3) Que los estudiantes desarrollen un compromiso ético en su configuración como profesionales, compromiso que debe potenciar la idea de educación integral, con actitudes críticas y responsables; garantizando la igualdad efectiva de mujeres y hombres, la igualdad de oportunidades, la accesibilidad universal de las personas con 
discapacidad y los valores propios de una cultura de la paz y de los valores democráticos.

De la misma manera, la asignatura Expresión y Comunicación Corporal pretende contribuir al desarrollo de las siguientes competencias específicas

(1) Conocer y comprender de manera fundamentada el potencial educativo de la Educación Física y el papel que desempeña en la sociedad actual, de modo que se desarrolle la capacidad de intervenir de forma autónoma y consciente en el contexto escolar y extraescolar al servicio de una ciudadanía constructiva y comprometida. Más concretamente: (a) Conocer y valorar el propio cuerpo y sus posibilidades motrices. (b). Conocer y dominar los fundamentos de la expresión corporal y la comunicación no verbal. (c). Conocer tipologías básicas de instalaciones y de material simbólico y funcional relacionados con la actividad física. (d). Conocer los aspectos que relacionan la actividad física con el ocio y la recreación para establecer las bases de utilización del tiempo libre: teatro, danza, deportes, salidas, etc.

(2) Saber transformar el conocimiento y la comprensión de la Educación Física en procesos de enseñanza y aprendizaje adecuados a las diversas e impredecibles realidades escolares en las que los maestros han de desarrollar su función docente. Más concretamente: (a) Dominar la teoría y la didáctica específica de la E.F., los fundamentos y las técnicas de programación del área y diseño de las sesiones, así como las estrategias de intervención y de evaluación de los resultados. R(b)acionar la actividad física con las distintas áreas que configuran el currículo de primaria, incidiendo en el desarrollo de la creatividad y las distintas manifestaciones expresivocomunicativas. (c) Orientar las actividades físicas que se desarrolla en el centro, en horario escolar y extraescolar, promoviendo la escuela como un entorno activo y saludable.

Se trata de una materia troncal de 6 créditos cuya práctica parte del principio de reciprocidad que permite establecer y mantener con los estudiantes una relación de mútua confianza. Por ello, dentro del proceso formativo, deben entrar en juego capacidades necesarias para el desarrollo personal y profesional como el diálogo, la escucha activa, el compromiso, la responsabilidad, la cooperación o la sinceridad, (Fraile, 2013). El nivel de confianza generado y sostenido será pilar esencial para el éxito o el fracaso en estas relaciones.

La organización de la asignatura se estructura en tres sesiones semanales de dos horas cada una, en las que se busca establecer una relación entre la teoría y la práctica. Inicialmente, se parte de un proceso de negociación del programa con los estudiantes en donde están presentes la finalidad de la asignatura, los contenidos, las actividades, la organización de los grupos, el método cooperativo de enseñanza y aprendizaje, los criterios de evaluación de los informes y el itinerario para llegar hasta las calificaciones finales.

Este desarrollo metodológico exige que el docente adopte un rol de facilitador e impulsor de dinámicas que ayuden a los estudiantes a mostrarse de forma auténtica, ayudándoles a sacar hacia fuera (exteriorizar) lo más genuino de su ser y su saber hacer.

En las tres semanas iniciales, el profesor parte de un tema de fundamentación teóricopráctica sobre los conceptos claves de la asignatura, las pautas para diseñar, aplicar y evaluarlas capacidades expresivas, y la presentación de los modelos y tendencias en la expresión corporal. Aquí, los estudiantes se familiarizan con el aprendizaje cooperativo y realizan los primeros trabajos grupales en relación a los conocimientos previos, conocimientos adquiridos, glosario terminológico del tema ydiseño de un plan de acción (objetivos, contenidos, 
actividades, criterios y pautas de evaluación, etc.) para poner en práctica una propuesta didáctica de expresión corporal destinada a la etapa de educación primaria.

La disposición de esta actividad formativa ayuda a que cada grupo a lo largo del desarrollo de la asignatura asuma dos funciones: (1) relacionada con la actividad docente (elaborar el contenido a desarrollar y a enseñar, presentar a nivel teórico-práctico tales contenidos y evaluar los informes que realizan semanalmente el resto de los grupos); y $\left(2^{\mathrm{a}}\right)$ específica como estudiantes, participando en el proceso cooperativo que culmina con la redacción de los informes semanales y finales (Fraile 2004, 2006) en relación a cómo se ha ido asentando personalmente la experiencia vivida y las competencias experimentadas y desarrolladas.

A lo largo del programa, los grupos trabajan los contenidos sobre las dimensiones corporales con la ayuda del profesorado, y van proyectando, llevando a la práctica y evaluando las distintas propuestas prácticas de expresión corporal, integrando los saberes teóricos, con los saberes prácticos y con el saber personal (ser o cómo estar). Es en la reflexion diaria a cerca del saber personal, en donde se contextualiza la aplicación del cuestionario de evaluación de las $\mathrm{Cl}$.

En relación a las dimensiones corporales estructurales objeto del proceso de enseñanzaaprendizaje del programa de expresión corporal, y cuya organización parte de considerar cuáles son las relaciones que se establecen entre el cuerpo con otros ámbitos motrices, se recogen a continuación los siguientes bloques de contenidos a tratar:

(1) El cuerpo se expresa en el espacio. Una de las finalidades de la expresión corporal para los estudiantes es poder tomar conciencia de su cuerpo desplazándose para ocupar espacios, siguiendo diferentes trayectorias, orientándose con respecto a los demás y a los objetos, y estructurando y organizando su entorno (Cratty, 1982). Se trata de explorar, interactuar en distintos espacios (propio, próximo y lejano), compartir, provocando que los estudiantes los distingan y los relacionen con tareas en las que expresen su emocionalidad.

(2) El cuerpo se expresa rítmicamente. Supone la toma de conciencia del tiempo a partir de los hechos que suceden durante un determinado tiempo. Para la adquisición de este concepto es clave el desarrollo del ritmo en cuanto la capacidad de organizar temporalmente estructuras regulares a reproducir mediante gestos o movimientos corporales (Fraisse, 1976). Los estudiantes, desde la vivencia de su ritmo corporal, muestran sus habilidades de imitación, creación e improvisación. Hacen uso de los conceptos de orden y duración temporal como base de las actividades expresivas rítmicas y coreográficas, que se pueden desarrollar a trave's de diversos componentes rítmicos (monorritmos, polirritmos, tareas arrítmicas o de improvisación) y empleando para ello algunos estilos musicales como el pop, rock, jazz, clásica, smooth o funky.

(3) El cuerpo se expresa para encontrar la felicidad. La perspectiva hedonista tiene como finalidad la búsqueda del movimiento expresivo, generando emociones que identifiquen estados de felicidad y placer. Para representar esta mirada del cuerpo, se adoptan los principios de la psicología positiva de Seligman (2014), cuya finalidad es fomentar un sentimiento optimista entre la ciudadanía.

Desde el punto de vista educativo esta ' presente el optimismo y el sentido del humor (risoterapia), que se presenta a los estudiantes desde el juego expresivo (García Larrauri, 2006).

(4) El cuerpo se comunica expresando. El ser humano, además de comunicarse con la palabra, lo hace también con el lenguaje corporal que representa un reflejo externo de la condición emocional de la persona (Pease \& Pease, 2006). La comunicación no verbal es una forma de interacción con los demás (Mata, Rodríguez, Diéguez y Bolivar, 2004) en la que la palabra se sustituye por otros códigos (kinéticos, el paralenguaje, la proxémica 0 la distancia entre los interlocutores). Desde esta perspectiva los gestos permiten la emisión de mensajes cargados de emociones, incluso con mayor significado que con la propia voz. Como 
componentes de esta manera de transmisión corporal, desde la asignatura, los participantes investigan y vivencian diversos tipos de expresión facial, gestual, miradas y posturas.

(5) El cuerpo se expresa creando e inventando. La creatividad es la capacidad de la persona para obtener algo de nada. Para Marina y Marina (2013), esa capacidad representa la posibilidad de dirigir las operaciones mentales normales, mediante un proyecto creador con el que se pretende alcanzar una antigua meta por caminos nuevos o mediante la búsqueda de una meta nueva. Para desarrollar esta competencia el profesor ha de convertirse en facilitador y guía, estimulando a los estudiantes a que expresen sus emociones y sentimientos; a propiciar una actividad que les ayude a sentirse libres para expresar las emociones, venciendo el miedo al error y observándole como un componente más del propio aprendizaje. La capacidad creativa se construye desde la actividad frente a la pasividad, desdela expresividad ante el mutismo, desde la innovación frente a la repetición y desde la independencia crítica frente a la sumisión intelectual.

(6) El cuerpo se expresa cooperando. El aprendizaje cooperativo implica un trabajo grupal a partir de una interacción simultánea y una participación equitativa. El compromiso de cada estudiante no es sólo consigo mismo, sino hacia el aprendizaje de sus compañeros (Fraile, López Pastor y Velázquez, 2014). Se hace presente así la competencia relacionada con las habilidades interpersonales y sociales que necesita, tal y como señalan Johnson y otros (1995)del desarrollo de capacidades comunicativas (tono de voz adecuado, evitar comentarios hirientes),operativas (encauzando la tarea, gestionando el tiempo, pidiendoy prestando ayuda), de comprensión profundade la tarea y los materiales (explicar, buscar recursos, resumir) y capacidades de razonamiento de alto nivel, argumentación y pensamiento crítico (discusión de ideas sin crítica hacia las personas, pedir opiniones justificadas). Desde el aprendizaje cooperativo, se favorece un proceso de enseñanza-aprendizaje donde los estudiantes aprenden juntos, se ayudan a través del intercambio de conocimientos y experiencias, y donde la evaluación formativa es clave para acentuar este tipo de aprendizajes.

(7) El Cuerpo se conoce a sí mismo y desde su interior se expresa. La actividad introyectiva forma parte de un proceso de búsqueda interna para el mejor autoconocimiento personal. Desde la introyección, la persona puede llegar a prestar más atención a su cuerpo tomando conciencia de las sensaciones más internas y pudiendo alcanzar un adecuado equilibrio y armonía interior (Rovira, 2010). La capacidad introyectiva de los estudiantes se desarrolla a través de actividades en las que se empleen técnicas que les permitan conseguir un mejor dominio de su sistema respiratorio (clavicular, torácico-bucal y abdominal-diafragmático), y técnicas como la relajación progresiva de Jacobson, el entrenamiento autógeno de Schultz, la reeducación psicotónica de Ajuriaguerra o la pedagogía de relajación de Alexander. Gracias a esa percepción introyectiva los estudiantes pueden ser más conscientes de sus vivencias motrices, considerando la conciencia como la integración de la experiencia expresivo-corporal.

(8) El cuerpo se expresa viviendo y transmitiendo emociones. Las emociones juegan un papel esencial en las relaciones interpersonales, ya que nos permiten afrontar diversos estados de ánimo en nuestras vivencias corporales diarias. Cada una de las emociones predispone de forma diferente a la acción, a veces dificultando y otras favoreciendo la capacidad de pensar, planificar o solucionar problemas (Goleman, 1998). La competencia emocional representa junto con las competencias mas funcionales un tándem esencial para el desarrollo profesional (Bisquerra y Pérez Escoda, 2007).

Salovey y Sluyter (1997) consideran que el desarrollo de la inteligencia emocional se va alcanzando a través de aspectos como: (a) la capacidad de reconocer las propias emociones como clave para reconocer y atender las emociones de los demás (alumnado, familias); (b) la capacidad de controlar las emociones y adecuarlas al momento: (c) la capacidad de motivarse a 
sí mismo; (d) el reconocimiento de las emociones ajenas (empatía); (e) el control de las relaciones (relacionarnos con las emociones ajenas).

Las actividades expresivas que se proponen a lo largo del programa ayudan a los estudiantes a mejorar su competencia personal, desde tareas que inciden en la autoconciencia (conciencia emocional de uno mismo, autovaloración y confianza en las propias posibilidades) y la autogestión de las emociones (iniciativa, optimismo, adaptabilidad, transparencia). Igualmente ayudan a mejorar su competencia social proponiendo actividades que les permitan reflexionar sobre su conciencia social (empatía, servicio, conciencia social) y la gestión que hacen de las interacciones que mantienen (liderazgo, gestión de conflictos, colaboración y trabajo en equipo, establecimiento de vínculos) (Goleman, Boyatzis y Mckee, 2002).

\subsection{Las competencias docentes}

El término competencia, mas allá de la óptica exclusivamente profesional, sugiere la habilidad para actuar de forma adecuada frente a los desafíos que situaciones y problemas plantean a los individuos, necesitando integrar para ello conocimientos complejos, habilidades y actitudes. Esto permite vislumbrar que el núcleo de una competencia está formado por aptitudes (saber y saber hacer) y actitudes (ser y saber estar), y que la resolución de problemas son su leitmotiv y su uso se relaciona con estándares social y profesionalmente establecidos (Escudero Muñoz, 2009).

Así, desde el punto de vista formativo,un docente de EF necesita no solo controlar y dominar los conocimientos y recursos del área, sino también aprender a pensar, a trabajar, a vivir y a ser, en el sentido armónico de confluencia entre saberes, comportamientos y habilidades en la vida de los individuos, aprendiendo además a actuar adaptativamente en los distintos contextos de manera reflexiva y sentida (Alberici y Serreri, 2005).

El Proyecto Tuning (Wagenaar \& González, 2003),reestructuró el marco formativo universitario en todo el territorio de la Unión Europea, y estableció el enfoque competencial en la formación universitaria, como mecanismo para garantizar la suficiente capacitación para la inserción profesional y el desempeño laboral eficiente. De la misma forma, la apuesta por las competencias en la nueva estructura educativa universitaria planteada (Espacio Europeo de Educación Superior), supuso un acercamiento a nuevas cotas de empleabilidad en un mercado de empleo cambiante y complejo, una apertura de fronteras de mercado, un mayor flujo de profesionales por el territorio europeo y una consideración al estudiante como centro de todo el proceso formativo.

El Proyecto Tuningtipifica las competencias en dos grandes grupos: Competencias Específicas, relacionadas con la posesión de conocimientos y habilidades especializadas en relación a un determinado campo laboral, convirtiendo al profesional en un experto que domina los contenidos y tareas propias para ejercer, en nuestro caso, la docencia de la EF (Echevarría, 2002), y Competencias Transversales (comunes en todas las titulaciones, profesiones y agrupadas en torno a tres subgrupos: competencias Instrumentales, Interpersonales y Sistémicas),

En relación a las Competencias Transversales, y dentro del subgrupo de las denominadas Interpersonales, se integran otras seiscompetencias que desarrollan diversascapacidades relacionadas con la inteligencia emocional y social (Goleman, 2006), que requieren del conocimiento y funcionamiento de las relaciones sociales y del comportamiento inteligente en ellas. De tal ejercicio, dependen importantes capacidades para la praxis docente como las habilidades comunicativas, la cooperación, la apertura hacia la diversidad o el dominio de las habilidades sociales.

Se muestran en la Figura 1, las seisCl, que tomamos a partir del Proyecto Tuning y que son 
objeto de exploración, vivencia y evaluación: (1) Trabajo en equipo; (2) Capacidad de comunicación interdisciplinar; (3) Habilidades en las relaciones interpersonales; (4) Apreciación de la diversidad y de la multiculturalidad; (5) Capacidad de autocrítica y crítica; (6) Compromiso ético.

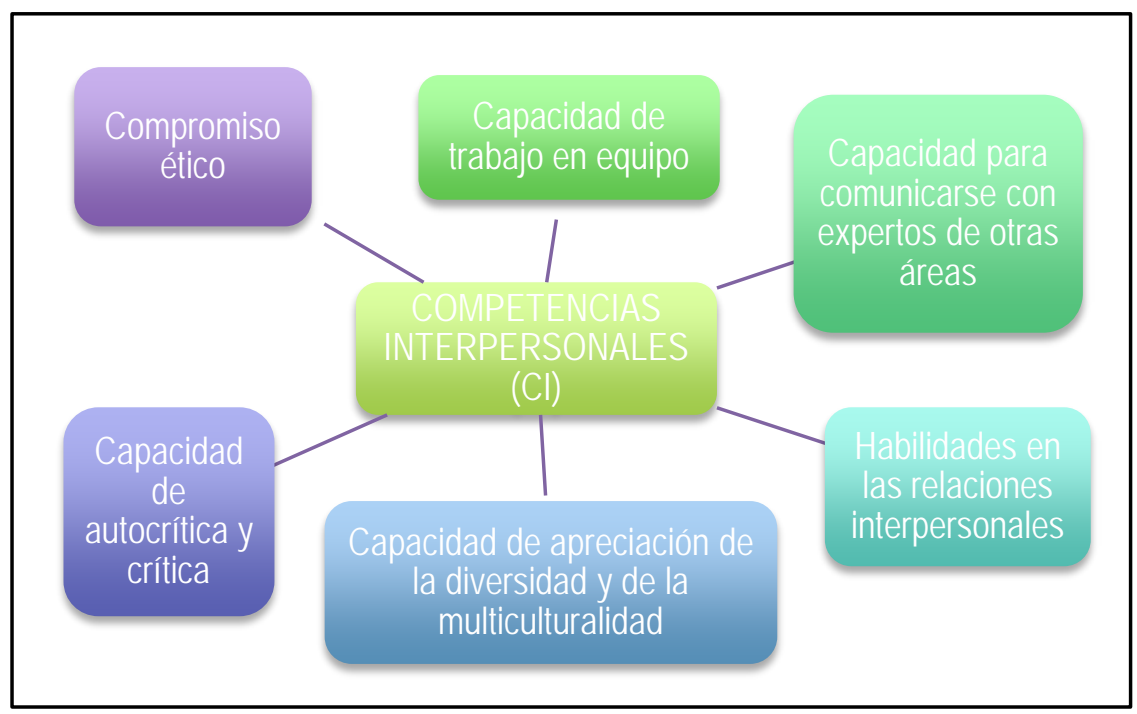

Figura 1: Competencias Transversales Interpersonales (Proyecto Tuning)

\subsection{Instrumentos de evaluación}

Durante el desarrollo del programa, y tras la reflexion y revision constante sobre el saber personal del profesorado y sus $\mathrm{Cl}$, se recoge información sobre el grado de adquisición y manejo de las mismasa través del cuestionario "Escala de valoración de competencias interpersonales del docente de Educación Física" (EcoiDEF) que se diseña a partir de cuestionarios similares de otras investigaciones que valoran las competencias genéricas de estudiantes universitarios (Solanes Puchol, Núñez Núñez, y Rodríguez Marín, 2008), y otros sobre competencias genéricas en contextos de docencia universitaria (Corominas et al., 2006).

Se estructura en 33 ítems en relación a las seis $\mathrm{Cl}$ objeto de exploración y evaluación, mediante indicadores que definen las capacidades constitutivas de tales competencias y a partir de la bibliografía especializada analizada.

Además se obtiene información desde el análisis de contenidos de 47 "Informes de autoevaluación sobre competencias interpersonales" correspondientes a los 47 estudiantes que cursan la asignatura. Tales informes se contextualizan en su formación como parte de la evaluación de la asignatura. En este sentido seles pide una reflexion final personal por escrito en donde valoren sus avances y retrocesos en relación a las vivencias sobre las $\mathrm{Cl}$ manejadas a lo largo del desarrollo del programa.

\section{Resultados}

Tras volcar las respuestas de los cuestionarios en una matriz en el paquete estadístico SPSS (v.21), se procedió al estudio de los datos mediante análisis estadístico descriptivo, hallando los valores medios de la distribución (medidas de tendencia central) y las medidas de dispersión, para obtener una idea sobre la representatividad de las medidas centrales. El estadístico de 
tendencia central utilizado es la media (M). Las medidas de dispersión nos ofrecen el grado de proximidad o lejanía que un conjunto de puntuaciones tienen entre sí. Utilizamos para ello la desviación típica $(\sigma)$.

La organización de la información proveniente del análisis de contenidos que se ha realizado a partir de categorías y subcategorías, no era lo suficientemente extenso como para hacer uso de instrumentos específicos para su análisis, siendo suficiente la elaboración de un cuadro para ordenar y categorizar las evidencias y citas, dada la extensión abarcable de los documentos manejados.

Para el análisis e interpretación de los resultados del cuestionario, hemos considerado atender al promedio de los valores aportados. Dado que no hemos obtenido promedios por debajo de 2,5, establecemos la siguiente valoración de resultados para su interpretación:

Entre 2,5 y 3,5 de media: nivel de valoración de competencias medio.

Entre 3,6 y 4,5 de media: un nivel de valoración de competencias alto

Entre 4,6 y 5 de media: nivel de valoración de competencias muy alto.

\subsection{Valoración general de las $\mathrm{Cl}$ para los estudiantes de Educación Física}

Tal y como se muestra en el Gráfico 1, las CTI más valoradas por los estudiantes son:

$1^{\text {a }}$. Compromiso ético

$2^{\mathrm{a}}$. Habilidades en las relaciones interpersonales

$3^{\mathrm{a}}$. Trabajo en equipo

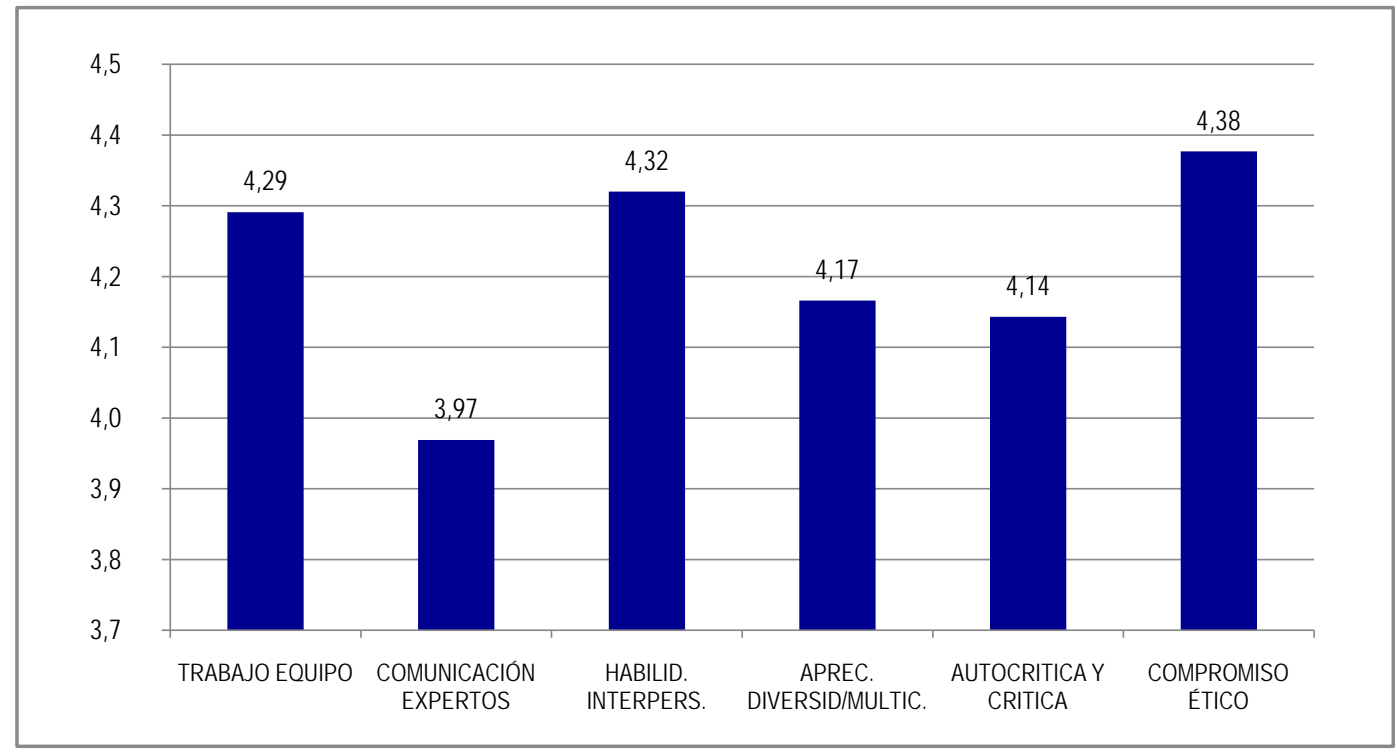

Gráfico 1: Representación gráfica de la valoración de las $\mathrm{Cl}$ analizadas por los estudiantes

3.2 Valoración particular de cada $\mathrm{Cl}$ y sus capacidades constitutivas mejor valoradas por los estudiantes. 
Se apuntan a continuación para cada una de las $\mathrm{Cl}$, las capacidades constitutivas que han ayudado más a que las valoren, adquieran y controlen a lo largo del desarrollo del programa.

(1) Compromiso ético (CE): de las capacidades estructuradorasdeesta competencia, a juicio de los estudiantes, destacan tres por encima del resto de las propuestas (Gráfico 2):

$1^{\mathrm{a}}$. Desarrollar actitud de respeto y tolerancia hacia los demás (CE5)

$2^{\mathrm{a}}$. Valorar y aceptar el punto de vista de los demás (CE1)

3a. Desarrollar una actitud más empática con los demás (CE4)

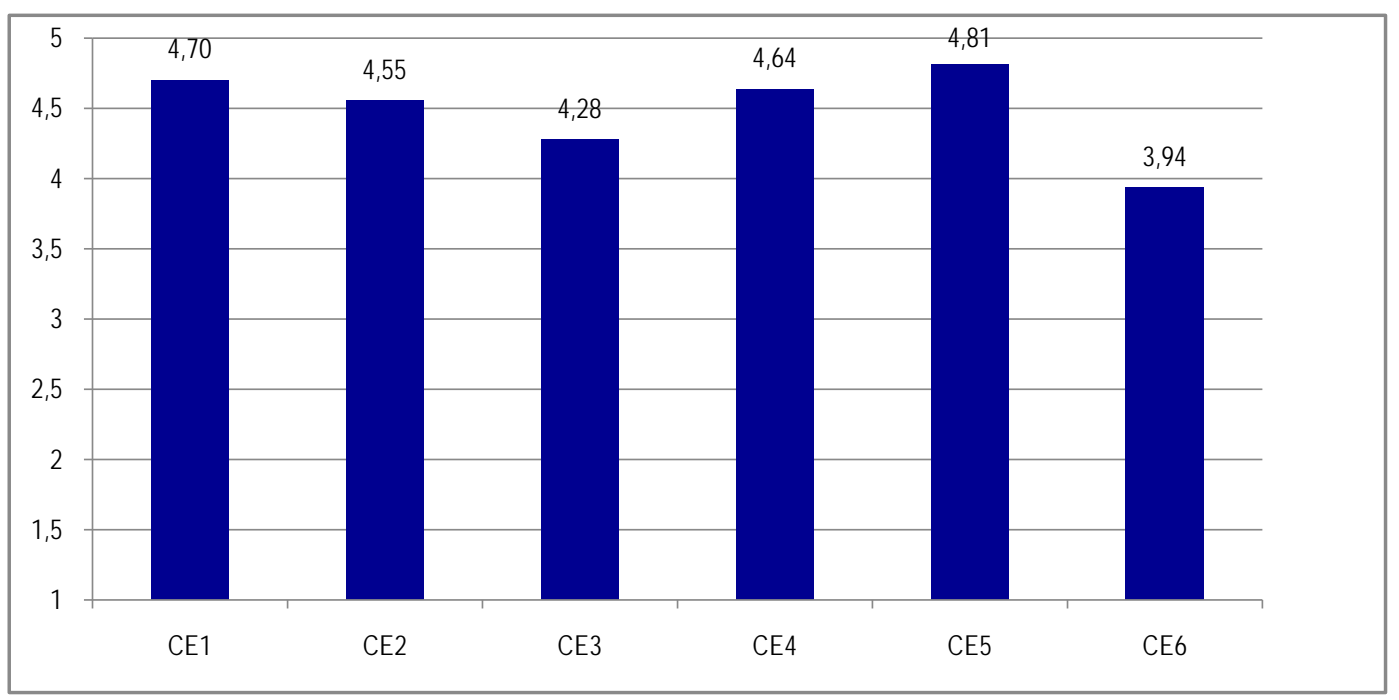

Gráfico 2: Representación gráfica de las capacidades que estructuran la competencia CE

Como se aprecia en el Gráfico 2, de las cinco capacidades que se consideraron vertebradoras del CE, la valoración más alta que hacen los estudiantes es hacia CE5 con un promedio de $M=4,81$ y un óptimo acuerdo de respuesta $(\sigma=0,4)$. En segundo lugar se valora CE1 con un promedio de 4,7 y una desviación de 0,5. Finalmente es reconocida la capacidad CE4 como tercera mejor valorada con un promedio de $M=4,64$ y un acuerdo de respuesta de $\sigma=0,5$.

(2) Habilidades en las relaciones interpersonales (HBRI): las capacidades consideradas como prioritariamente constitutivas son las siguientes (Gráfico 3):

$1^{\text {a }}$. Mostrar un trato amable, respetuoso y responsable con los demás (HBRI3)

$2^{\mathrm{a}}$. Reconocer las preocupaciones, intereses y necesidades de los demás (HBRI1)

$3^{\text {a }}$. Valorar las emociones y sentimientos de los demás (HBRI4) 


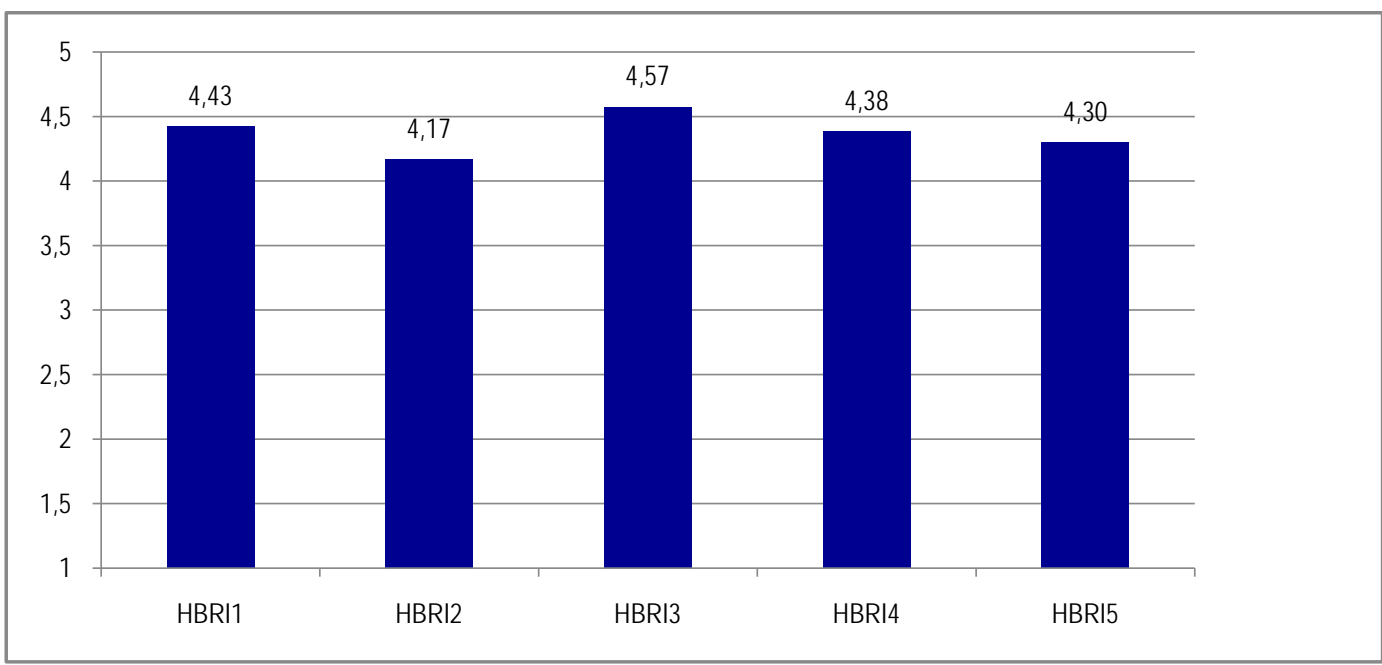

Gráfico 3: Representación gráfica de las capacidades que estructuran la competencia HBRI

De las cinco capacidades que se consideraron vertebradoras de HBRI, la más valoradas por los estudiantes es HBRI3 obteniendo un promedio común de $\mathrm{M}=4,57$ y un acuerdo de respuesta similar de $\sigma=0,5$. En segundo y tercer lugar, las capacidades mejor posicionadas son HBR1 y HBR4 con un promedio de $M=4,43$ y $M=4,38$ y con acuerdos de respuesta similares de $\sigma=0,6$ y $\sigma=0,7$ respectivamente.

(3) Trabajo en equipo (TE): En el Gráfico 4 se muestra la preferencia por tres capacidades estructuradoras del TE:

$1^{\mathrm{a}}$. Mostrar predisposición a la colaboración (TE3)

$2^{\text {a }}$. Manifestar capacidad de escucha activa (TE4)

$3^{\text {a }}$. Compartir ideas proyectos y materiales (TE1)

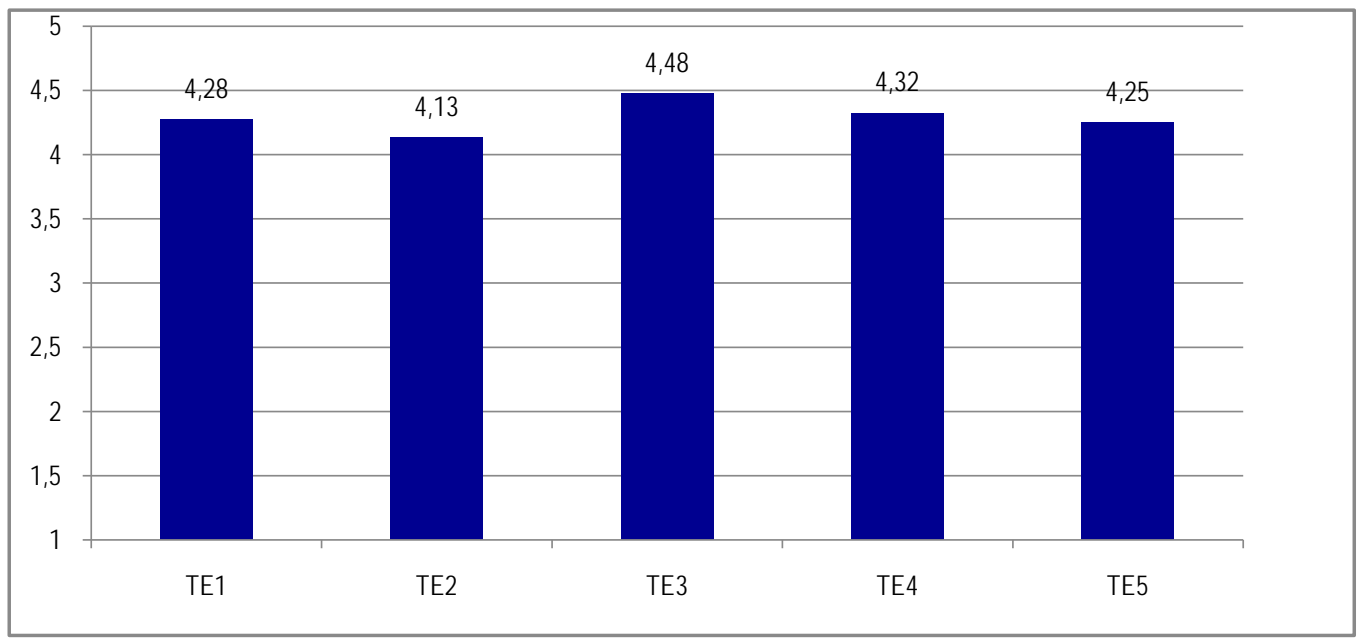

Gráfico 4: Representación gráfica de las capacidades que estructuran la competencia TE 
Finalmente, son tres las capacidades constitutivas de la competencia TE, que mejor valoran los estudiantes para su adquisición y desarrollo. Para TE3 se establece un promedio de $M=4,48$ y una desviación típica de $\sigma=0,6$. Por su parte TE4 obtiene una valoración media de $\mathrm{M}=4,32$ y un óptimo acuerdo de respuesta igualmente de $\sigma=0,6$. La capacidad TE1 obtiene una valoración de promedio de $M=4,28$ y un acuerdo de respuesta de $\sigma=0,7$.

\section{Conclusiones}

En relación al compromiso ético, la valoración de sus capacidades constitutivas, y a la luz de las evidencias sobre el análisis documental de los informes de evaluación, se evidencia que el itinerario de apertura a la competencia se iniciacon la valoración de la importancia de desarrollar una base empática personal, sobre la que construir con el alumnado un estilo de convivencia en el aula responsable y comprometido con los problemas que atraviesan la vida del aula y del centro (Fraile y Aparicio, 2014). En el comportamiento y actitudes del docente deben primar el respeto, la cercanía, la empatía, la responsabilidad, la actitud positiva y la implicación. Un perfil docente ejemplar cuya ética profesional, honradez, compromiso y coherencia interna son indispensables para una docencia responsable, destacando en él la vivencia de valores democráticos y cívicos, coincidiendo esta valoración con lo recogido en el Informe Delors (1996).

Para los estudiantes, la capacidad de compromiso ético como docentes de educación física, supone adoptar un auténtico posicionamiento democrático para reconocer y valorar los esfuerzos de la comunidad educativa (alumnado, madres, padres, profesorado) para organizar la vida de los centros, y facilitar así el proceso de enseñanza y aprendizaje y la atención a cuantas problemáticas atraviesan la vida de los centros y del alumnado y sus familias. El compromiso ético,en este sentido, require de un esfuerzo en la búsqueda de soluciones conjuntas, lo que posibilita la union efectiva de educación y democracia para concebir mejores individuos sociales que se sensibilizan y responsabilizan ante los cambios y los problemas sociales (De Juanaset al., 2010).

Es crucial para adoptar un rol de educador democrático, respetar las opiniones de todos, dispensando un trato digno hacia el alumnado, las familias y el profesorado, principios educativos que subyacen de la reflexion y valoración de los estudiantes y coincidente con los estudios de Fernández Balboa, 2000.

En lo referente al trabajo en equipo, los estudiantes manifiestan una disposición positiva a aceptar la diversidad de formas de pensar, a adaptarse a las características personales de los miembros que conforman el equipo, a la variada tipología de personas que podrían formar parte de dichos equipos, a compartir ideas, proyectos, materiales y retos para contribuir a la configuración de un óptimo clima de trabajo cooperativo. Se reconoce que el trabajo en equipo requiere de competencias personales, sociales y actitudes abiertamente inclusivas para desarrollar una EF cooperativa, coincidiendo en ello con los planteamientos de Fraile, López Pastor y Velázquez (2014), y con la visión cooperativa de las competencias relacionadas con la organización, la planificación y la toma de decisiones conjunta para resolver problemas, mostrada en los trabajos de Pazo Haro y Tejada Mora (2012) con estudiantes de Educación Física para la autopercepción de sus competencias transversales.

Los nuevos modos de profesionalidad docente implican un incremento de las dimensiones colectivas y de colaboración, de la intervención conjunta en los proyectos educativos, de la ayuda mútua y de la capacidad de compromiso de cada estudiante para consigo mismo y para con el aprendizaje de sus compañeros, lo que trae consigo un esfuerzo de responsabilidad compartida durante el desarrollo de la dinámica metodológica del programa de expresión corporal. La autopercepción más cooperativa del trabajo en equipo desempeñado a lo largo del programa, evidencia una clara priorización por esta competencia docente y su anclaje en el 
bagaje formativo de los estudiantes. Una experiencia similarde Fraile (2009), con estudiantes de educación física,evidencian esta percepción y en este mismo sentido.

Para ello, el ejercicio profesional se debe organizar, cada vez más, en torno no solo a comunidades de práctica en el seno del aula, sino también en el contexto de movimientos pedagógicos conectados a dinámicas que vayan más allá de las fronteras organizativas y del propio centro escolar (comunidades de aprendizaje).

Finalmente en relación a las habilidades en las relaciones interpersonales, los estudiantes muestran la empatía como capacidad preferentey a quien otorgan suma importancia (al hablar de gestión de las emociones frecuentemente aparece el término), junto con el reconocimiento de los propios sentimientos y emociones, como claves para enfrentarse a las relaciones personales con los demás y, por tanto, de obligada presencia en las relaciones profesionales, y como aspecto esencial para comprender y actuar sobre las emociones del alumnado. Esta disposición por "meterse en la piel" del alumnado para ayudarle más y mejor, también se refleja en los trabajos de Martínez, Romero, Romero y Zagalaz (2011).

De esta forma, ser consciente, entender y apreciar los sentimientos de los demás (la empatía) es clave para la vivencia de las relaciones interpersonales y para construir adecuadas habilidades comunicativas y de flexibilización de la espera hacia el alumnado que mas lo necesita, o por el contrario de ajuste en los requerimientos de aquel alumnado cuyo ritmo es más rápido. En este sentido coincidimos con los resultados obtenidos sobre competencias emocionales en los trabajos con estudiantes de magisterio de Abarca y Sala (2002).

Se evidencia por tanto, que el dominio de las $\mathrm{Cl}$ les puede ayudar a afrontar adecuadamente los imperativos prácticos de la vida, lo que va a permitir en la praxis docente no solo contar con los conocimientos propios del área de educación física como única herramienta de intervención, sino con diversas capacidades que les permitan manejar adecuadamente las relaciones con el alumnado, sus familias, el profesorado y otros agentes socioeducativos, estableciendo negociaciones, creando un clima adecuado de aula y centro, valorando lasvivencias, emociones y sentimientos, estableciendo relaciones productivas de colaboración,y contribuyendo con ello a acercar las actividades físicas a prácticas educativas mas humanizadoras (Ruiz Omeñaca, 2004), creativas y sentidas (Gracia, Rodríguez Jiménez y Terrón, 2010).

\section{Referencias bibliográficas}

Abarca, M., y Sala, J., (2002). Las competencias emocionales de los futuros profesores. Revista Electrónica Interuniversitaria de Formación del profesorado, 3 (5), pp. 1-4.

Alberici, A., y Serreri, P. (2005). Competencias y formación en la edad adulta. Balance de competencias. Barcelona: Alertes.

Bisquerra Alzina, R., y Pérez Escoda, N. (2007). Las competencias emocionales. Educación XX1(10), pp. 61-82.

Bolívar, A. (2010). Contexto de la Educación Secundaria: Estructura y Organización. En Imbernón (coord.), Procesos y Contextos Educativos: Enseñar en las instituciones de Educación Secundaria (pp. 35-54). Barcelona: Graó.

Cratty, B.J. (1982). Desarrollo perceptual y motor en los niños. Barcelona: Paidós.

Corominas, E., Tesauro, M., Capell, D., Teisidó, J., Pèlach, J., y Cortada, R. (2006). Percepciones del profesorado ante la incorporación de las competencias genéricas en la formación universitaria. Revista de Educación (341), pp. 301-336. 
De Juanas, A., Fernández, P., González, M., Martín del Pozo, R., Sánchez, M., y Pesquero, E. (2010). Las competencias de los maestros de primaria: percepción de los estudiantes en prácticas. Investigación en la escuela 72, pp. 71-83.

Delors, J., y otros (1996): La educación encierra un tesoro. Informe a la UNESCO de la Comisión Internacional sobre la Educación para el Siglo XXI. Madrid. Santillana/UNESCO.

Echevarría, B. (2002). Gestión de la competencia de acción profesional. Barcelona: Universitat de Barcelona.

Escudero Muñoz, J. (2009). Las competencias profesionales y la formación universitaria: posibilidades y riesgos. Revista interuniversitaria de pedagogía social 16, pp. 65-82.

Fernández Balboa, J. M. (2000). La Educación Física para el future: tendencias sociales y premisas educativo-profesionales. Tandem, 1, pp. 15-26.

Fraisse, P. (1976). Psicología del ritmo. Madrid: Morata.

Fraile, A. (2004): Un cambio democrático en las aulas universitarias: Una experiencia en la formación del profesorado de Educación Física. Contextos educativos: Revista de Educación, núm. 6-7, pp. 213-234.

Fraile, A. (2006): Cambios en el aula universitaria ante los nuevos retos europeos. Tándem. Didáctica de la Educación Física, núm. 20, pp. 57-72

Fraile, A. (2009). Generic competences in the physical education teacher training. Studia Universitatis Babes-Bolyai, Educatio Artos Gimnasticae, 1 (54), pp. 9-16.

Fraile, A. (2013): El coaching como estrategia pedagógica para la mejora del autoconocimiento del educador deportivo. Revista de Educación, Motricidad e Investigación, 1, pp.1-13.

Fraile, A. y Aparicio, J.L. (2014). Competencias interpersonales que deben estar presentes en la formación inicial del profesorado de educación física. Acción, 21 (11), pp. 25-29.

Fraile, A., López Pastor, V., y Velázquez, C. (2014). Aprendizaje Cooperativo en Educación Física. Movimento, 20(1), pp. 239-259.

García Larrauri, B. (2006). Programa para mejora el sentido del humor. Madrid: Pirámide.

Gracia, P., Rodríguez Jiménez, R.M. y Terrón, M.J. (2010). Recursos creativos corporales y uso de la comunicación no verbal para el desarrollo y la evaluación de competencias genéricas en alumnos de grado. Revista de Docencia Universitaria, 1 (8), pp. 142-157.

Goleman, D. (1998). Inteligencia emocional. Barcelona. Kairós.

Goleman, D., Boyatzis, R. y McKee, A. (2002). El líder resonante crea más. Barcelona: Plaza y Janés.

Goleman, D. (2006). Inteligencia social. La nueva ciencia de las relaciones humanas. Barcelona: Kairós.

Johnson, D.W.; Johnson, R.T., y Holubec, E.J. (1995). Los nuevos círculos de aprendizaje. Alexandria VA:ASCD.

Marina, J.A. y Marina, E. (2013). El aprendizaje de la creatividad. Madrid: Ariel.

Martínez, E., Romero, C., Romero, M., y Zagalaz (2011). Importancia de las competencias profesionales de los Maestros de Educación Física expresadas por los estudiantes. Retos, Nuevas tendencias en Educación Física, Deporte y Recreación 19,pp. 62-68. 
Montálvez, M. y González López, I. (2012). La expresión corporal en educación física en primaria. Conclusiones sólidas para una sociedad líquida. Tándem. Didáctica de la Educación Física,39, pp. 8-21.

Ojiro, Y. Y Miralles, F. (2006). El Zen de la empresa. Barcelona: Alienta.

Ortega, R. (2002). Lo mejor y peor de las redes de iguales: Juego, conflicto y violencia. Revista Interuniversitaria de Formación del Profesorado, 44, pp. 93-113.

Pazo Haro, C.I. y Tejada Mora, J. (2012). Las competencias profesionales en Educación Física. Retos. Nuevas tendencias en Educación Física, Deporte y Recreación, 22, pp. 5-8.

Pease, A. y Pease, B. (2006). El lenguaje del cuerpo. Barcelona: Amat Editorial.

Rovira, G. (2010). La conciencia sensitiva en la formación docente. Estudio de caso: Las vivencias de los estudiantes de magisterio suscitados en la práctica de situaciones motrices introyectivas. Tesis doctoral. Universidad de Lleida.

Ruiz Omeñaca, J. (2004). Las actividades física cooperativas: una oportunidad para ampliar el valor educativo de nuestra área curricular. Tándem. Didáctica de la Educación Física 14, pp. 33-43.

Salovey, P. \& Sluyter, D. (Eds). (1997). Emotional Development and Emotional Intelligence: Implications for Educators. New York: Basic Books.

Seligman, M. (2014). Aprenda optimismo. Madrid: De Bolsillo.

Solanes Puchol, A., Núñez Núñez, R., y Rodríguez Marín, J. (2008). Elaboación de un cuestionario para la valoración de competencias genéricas en estudiantes universitarios. Apuntes de Psicología, 26 (1), pp. 35-49.

Talvio, M.; Lonka, K.; Komulainen, E., Kuusela, M. y Lintunen, T. (2013). Un nueva mirada a la formación en eficacia docente de Gordona (TET): Un estudio-intervención en el aprendizaje social y emocional del profesorado. Electronica Journal of Research in Educational Psychology, 11 (3), pp. 693-716.

Vaello, J. (2009). El profesor emocionalmente competente. Barcelona. Graó.

Vygotsky, L. S. (1978) Pensamiento y lenguaje. Madrid: Paidós.

Wagenaar, R., y González, J. (2003). Tuning Educational Structures in Europe. Informe Final. Fase uno. Universidad de Deusto. 